\title{
甲酸分解制氢均相催化剂的研究进展
}

\author{
刘嘉豪韩静杰易小艺刘超何 飘* \\ (中南大学化学化工学院 长沙 410083)
}

\begin{abstract}
摘要 甲酸 $(\mathrm{HCOOH})$ 具有作为液氢储存材料和清洁制氢的巨大潜力. 在过去的十几年中, 人们不仅在开发活性更强、 更稳定、选择性更强的催化剂方面取得了重大进展，而且提出了氢气释放和储存循环系统的概念. 综述了均相催化剂 在这一领域的最新进展, 重点介绍了钉、铑、铱为代表的贵金属催化剂, 同时还涉及铁、钴、镍、铜、铝等非贵金属 催化剂. 希望本综述对今后开发更好的甲酸脱氢催化体系提供一些见解和思路.
\end{abstract}

关键词 氢能源; 甲酸脱氢; 均相催化; 金属催化剂

\section{Research Progress of Homogeneous Catalyst for the Dehydrogenation of Formic Acid}

\author{
Liu, Jiahao Han, Jingjie Yi, Xiaoyi Liu, Chao He, Piao* \\ (College of Chemistry and Chemical Engineering, Central South University, Changsha 410083)
}

\begin{abstract}
Formic acid $(\mathrm{HCOOH})$ has great potential as liquid hydrogen storage materials and for clean hydrogen production. In the past decade, researchers have not only made significant progress in developing more active, stable, and selective catalysts, but also proposed the concept of the reversible system of hydrogen release and storage. This review covers the latest progress of homogeneous catalysts in this field, focusing on the noble metal based catalysts represented by ruthenium, rhodium, and iridium, as well as the non-noble metal based catalysts such as iron, cobalt, nickel, copper, aluminum and so on. It is hoped that this review will provide some insights and idea for developing better catalytic systems for the dehydrogenation of formic acid in the future.

Keywords hydrogen energy; formic acid dehydrogenation; homogeneous catalysis; metal catalyst
\end{abstract}

随着社会经济的发展及人类环保意识的日益增强, 人们开始寻找新的能源以代替现有的石化燃料. 氢能具 有清洁、高效、安全和可持续等优点, 将成为本世纪极 具发展潜力的能源. 我国经济高速增长、能源进口依赖 较大、消耗与日俱增, 因此, 氢能源的开发和利用具有 十分重要的战略意义. 一个以氢为基础的能源体系包括 氢气的生产、储藏、运输及转化应用等一系列环节, 其 关键在于氢气的生产与败存问题. 一方面, 目前工业制 备氢气的主要方法有化石燃料制氢、电解水制氢和催化 热分解碳氢化合物制氢. 以上这些方法, 都存在能耗过 高等缺点. 另一方面, 氢气存储的方法有高压/液化、多 孔材料吸附(分子篮、金属有机材料以及高分子聚合物 等)、化学储氢(金属氢化物、嗍氢化合物、有机小分子 等), 但由于条件严苛而带来成本过高的问题. 所以, 在
温和的条件下实现氢气的制备和存储具有重要的科学 意义和应用前景.

\section{1 甲酸储氢}

甲酸 $(\mathrm{HCOOH}, \mathrm{FA})$ 是最简单的一元有机羧酸, 具有 较高的储氢含量 $(w=4.4 \%, 53 \mathrm{~g} / \mathrm{L})$, 在合适的催化剂作 用下, 室温下即可分解为 $\mathrm{CO}_{2}$ 和 $\mathrm{H}_{2}$. 甲酸价格低廉、来 源广泛、毒性较低、常温常压是液态、性质稳定、不易 燃烧、运输和储存安全方便, 作为一种极具潜力的化学 储氢材料而被广泛研究 ${ }^{[1]}$. 甲酸分解主要通过两种反应 路径(图 1): (1)分解为 $\mathrm{CO}_{2}$ 和 $\mathrm{H}_{2}$; (2) 分解为 $\mathrm{CO}$ 和 $\mathrm{H}_{2} \mathrm{O}$. 两个反应在标准状态下均为自发反应, 但动力学受阻. 而且 CO 会使燃料电池中毒, 因此, 甲酸分解的研究主 要集中在开发低温下工作的高稳定性、高选择性、高活

\footnotetext{
* Corresponding author. E-mail: piaohe@csu.edu.cn

Received March 27, 2020; revised May 9, 2020; published online June 11, 2020.

Project supported by the Research Start-Up Fund of Central South University (No. 201810).

中南大学科研启动基金(No. 201810)资助项目.
} 
性的制氢催化剂, 使甲酸的分解过程按照式(1)的途径 进行. 此外, 甲酸和二氧化碳在储氢系统中的结合是一 种非常高效的储氢途径, 即可以通过还原 $\mathrm{CO}_{2}$ 生成甲酸 以存储氢气, 又可以适当分解甲酸释放氢气, 从而实现 氢能源的循环利用.

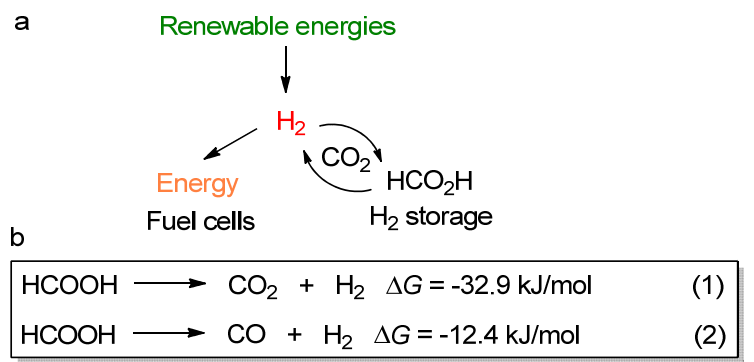

图 1 (a) $\mathrm{HCOOH}$ 和 $\mathrm{CO}_{2}$ 的循环系统以及(b) 甲酸分解的两种 路径

Figure 1 (a) $\mathrm{HCOOH}$ and $\mathrm{CO}_{2}$ circulation system, and (b) two paths for formic acid decomposition

\section{2 均相催化剂}

甲酸脱氢催化剂主要包括异相催化剂和均相催化 剂. 其中, 异相催化剂(贵金属、金属氧化物、碳负载金 属催化剂等)具有稳定性好、容易制备、易于分离、可 重复使用和回收等优点, 但催化剂的活性低[以转换频 率(TOF)来计算], 选择性复杂, 产生氢气的速度较慢. 均相催化剂, 主要结构单一, 选择性比较好, 反应产生 的 CO 体积分数一般在 $0.01 \%$ 以下. 均相催化剂自 1967

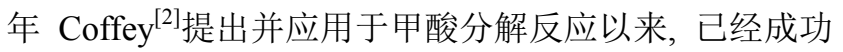
制备了 Pt、Ru、Ir 磷配位催化剂. 随后, 人们发展了一 系列以 $\mathrm{Ru} 、 \mathrm{Rh}$ 和 $\mathrm{Ir}$ 等过渡金属为中心, 以含膦化合物 (苯基膦磺酸盐、苄基膦、苯基膦等)、含氮化合物(联吡 啶、联嘧啶、二羟基联吡啶、邻二氮杂菲等)为配体的 金属络合物作为甲酸脱氢催化剂, 取得突破性进展.

\section{1 钉金属络合物}

1998 年, Puddephatt 等 $^{[3]}$ 报道了双核钓膦络合物 $\left[\mathrm{Ru}_{2}(\mu \text {-CO)(CO) })_{4}(\mu \text {-dppm })_{2}\right] \quad\left(\mathbf{1}, \mathrm{dppm}=\mathrm{Ph}_{2} \mathrm{PCH}_{2} \mathrm{PPh}_{2}\right)$, 在没有碱的情况下, 室温下以 TOF $500 \mathrm{~h}^{-1}$ 的初始速率 从甲酸中快速产生氢气, 并且活性随着胺的加入而增 加. 同年, $\mathrm{Gao}$ 等 ${ }^{[4]}$ 利用核磁共振波谱对各种中间氢化物 和甲酸盐络合物进行了鉴定, 成功分离出了 $\left[\mathrm{Ru}_{2} \mathrm{H}(\mu\right.$ $\mathrm{H})(\mu$-CO $\left.)(\mathrm{CO})_{2}(\mu \text {-dppm })_{2}\right]$ (2). 通过核磁检测该物质在 丙酮 $\left(5 \times 10^{-3} \mathrm{~mol} \cdot \mathrm{L}^{-1}\right)$ 与甲酸 $\left(10^{-1} \mathrm{~mol} \cdot \mathrm{L}^{-1}\right)$ 的密闭管内 反应, $20{ }^{\circ} \mathrm{C}$ 下在 $0.3 \mathrm{~h}$ 内完成, 平均转换数 $(\mathrm{TON})$ 约为 $70 \mathrm{~h}^{-1}$. 随后, Wills 等 ${ }^{[5-7]}$ 在以往转移氢化经验的基础上, 研究了三乙胺存在下钉催化剂对于甲酸脱氢反应的催 化性能. 其中, $\mathrm{RuCl}_{2}(\mathrm{DMSO})_{4} 、 \mathrm{RuCl}_{2}\left(\mathrm{NH}_{3}\right)_{6}$ 和无水 $\mathrm{RuCl}_{3}$ 在 $120{ }^{\circ} \mathrm{C}$ 下 4 次循环后, 表现出相同的活性, TOF
高达 $18000 \mathrm{~h}^{-1[8]}$. 由于在前三个循环中观察到活性的增 加, 推测在反应温度大于 $100{ }^{\circ} \mathrm{C}$ 的活化过程中, 形成了 常见的双核 $\mathrm{Ru}_{2}\left(\mathrm{HCO}_{2}\right)_{2}(\mathrm{CO})_{4}$ 络合物(3). 但反应温度升 高存在一个缺点: 甲酸脱羰基化的发生导致 $\mathrm{CO}$ 的形成 (体积分数为 $0.02 \%$ ), 这将阻碍其与燃料电池的长期耦 合. 为了保持稳定的催化活性，建立了连续的制氢装置, 可用高沸点的二甲胺取代三乙胺作为添加剂 ${ }^{[9]}$. 根据温 度或阻抗反馈机制, 可调整甲酸的用量以达到稳定(最 大)的氢气输出, 但仍会产生 CO.
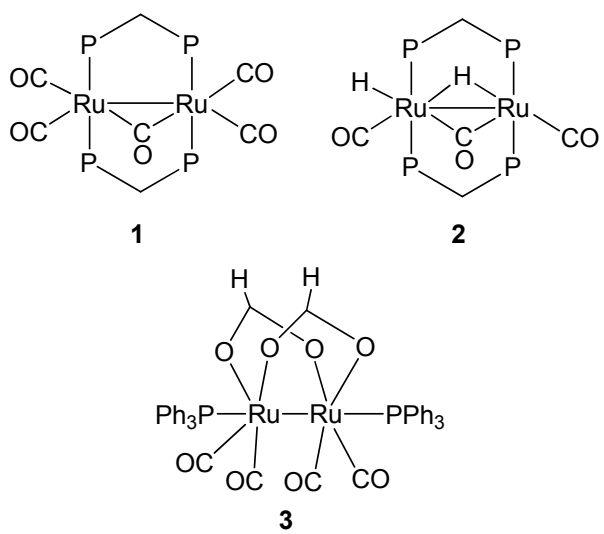

图 2 Puddephatt 和 Wills 报道的双核钉膦络合物 Figure 2 Binuclear ruthenium phosphate complexes reported by Puddephatt and Wills

在过去几十年的研究中, 甲酸一直被定义为转移氢 化反应中的氢供体, 而不是储氢介质. 直到 2008 年, 甲 酸作为液态有机氢载体的潜在应用研究不断深入, 甲酸 和甲酸盐的脱氢催化剂的活性和稳定性都得到了提高. 人们研究了添加物(醇、卤化物、水)、胺配体、膦配体、 光和溶剂对钉前体原位催化性能的影响, 结果表明碱强 度与催化剂活性有较强的相关性.

2008 年以来, Laurenczy 和同事 ${ }^{[10]}$ 开发了一种亲水 性均相催化体系 4 , 可以选择性地将甲酸分解成氢气和 二氧化碳. 他们报道了含有微量甲酸盐的甲酸水溶液与 $\left[\mathrm{Ru}\left(\mathrm{H}_{2} \mathrm{O}\right)_{6}\right](\mathrm{tos})_{2}$ (tos=甲苯-4-磺酸盐)在大范围的温度 $\left(70 \sim 120{ }^{\circ} \mathrm{C}\right)$ 和压力 $(1 \sim 22.0 \mathrm{MPa})$ 下可进行有效分解, 其中甲酸盐起到关键作用. 随着温度的升高, 反应速率 增大 $\left(120{ }^{\circ} \mathrm{C}\right.$ 时 $\left.\mathrm{TOF}=670 \mathrm{~h}^{-1}\right)$, 并且所有反应的转化率 都高于 $90 \%$. 当 $\left[\mathrm{Ru}\left(\mathrm{H}_{2} \mathrm{O}\right)_{6}\right](\operatorname{tos})_{2}$ 作为催化剂前体时, 需 要一个诱导期才能形成活性组分, 催化剂在温度达到 $170{ }^{\circ} \mathrm{C}$ 以及在溶液中放置一年后仍然表现出显著的稳 定性. 核磁共振谱研究表明, 反应机理由两个相互竞争 的循环组成, 通过一种具有两个顺式膦配体的氢化钓中 间体连接 ${ }^{[11]}$. 在连续的甲酸脱氢装置中, $120{ }^{\circ} \mathrm{C}$ 下 $90 \mathrm{~h}$ 内 TON 可达到 40000 以上，且没有检测到一氧化碳的生 成. 在 $\mathrm{RuCl}_{3} \cdot x \mathrm{H}_{2} \mathrm{O} / m \mathrm{TPPTS}$ 存在的情况下, 在第一个循 环中观察到初始的甲酸脱氢速率较高, 几次运行后最终 
活性与 $\mathrm{Ru}(\mathrm{II})$ 前体相当. 动力学研究表明, 三膦钓参与 了快速循环, 而双膦络合物被确定为较慢的循环部 分 ${ }^{[12]}$. 一系列具有给电子/吸电子和亲水性的磺化芳基 与 $\mathrm{RuCl}_{3} \cdot x \mathrm{H}_{2} \mathrm{O}$ 结合形成了高稳定和活性的催化剂体系, 在 $90{ }^{\circ} \mathrm{C}$ 的 $10 \mathrm{~mol} \cdot \mathrm{L}^{-1} \mathrm{HCOOH} / \mathrm{NaOOCH}(m: m=9: 1)$ 溶液中, TOF 达到 $476 \mathrm{~h}^{-1[13]}$. 在其它条件都相同的情况 下，120 ${ }^{\circ} \mathrm{C}$ 下亲水性氨甲基化三芳基膦的存在进一步提 高了催化活性 $\left(\mathrm{TOF}=1950 \mathrm{~h}^{-1}\right)^{[14]}$.

自 2008 年以来, Beller 和他的同事对甲酸分解催化 剂开展了详细研究, 报道了在 $40{ }^{\circ} \mathrm{C}$ 和有胺添加剂的条 件下，不同均相催化剂包括 $\mathrm{RuBr}_{3} \bullet x \mathrm{H}_{2} \mathrm{O}$ 、 $\left[\left\{\mathrm{RuCl}_{2}-\right.\right.$ (p-cymene) $\left.\left.\}_{2}\right] 、\left[\mathrm{RuCl}_{2}\left(\mathrm{PPh}_{3}\right)_{3}\right] 、\left[\left\{\mathrm{RuCl}_{2} \text { (benzene }\right)_{2}\right\}_{2}\right]$ 等 甲酸脱氢反应. 以 $0.1 \%\left[\left\{\mathrm{RuCl}_{2}(p \text {-cymene })\right\}_{2}\right]$ 为标准催 化剂前体, 甲酸与胺的比例为 $5: 2$, 探究了不同胺类对 催化活性的影响. 在 $N, N$-二甲基甲酰胺 (DMF) 和 FA/NEt ${ }_{3}(m: m=5: 2)$ 下催化活性最高的两种催化剂 $\left[\left(\mathrm{RuCl}_{2}\left(\mathrm{PPh}_{3}\right)_{3}\right] \quad\left(\mathrm{TOF}=445 \mathrm{~h}^{-1}\right)^{[15]}\right.$ 和 $\left[\mathrm{RuCl}_{2}\left(\mathrm{C}_{6} \mathrm{H}_{6}\right)_{2} /\right.$ DPPE] (5) $\left(T O F=460 \mathrm{~h}^{-1}\right)^{[16]}$. 为了避免在连续制氢过 程中胺的排放而导致催化失活, 使用 $N, N$-二甲基己胺替 代挥发性三乙胺，在室温下，使用优化的 $\left[\mathrm{RuCl}_{2}\left(\mathrm{C}_{6} \mathrm{H}_{6}\right)\right]_{2} / \mathrm{DPPE}$ 催化剂可以高效稳定地催化甲酸 产氢, TON 大约 260000, TOF 超过 $900 \mathrm{~h}^{-1[17-18]}$. 此外, 他们首次发现甲酸与钉前体的催化体系在光照射下的 活性增加 1 个数量级, 例如, 采用 $\left[\mathrm{RuCl}_{2}\left(\mathrm{C}_{6} \mathrm{H}_{6}\right)_{2} / \mathrm{DPPE}\right]$ 催化剂时, TON 从 407 增加到 2804 , 几乎增加了 7 倍. 这 是由于光诱导了钓芳烃络合物的活化, 以及光促进了活 性催化剂的再生 ${ }^{[19]}$. 近期, Beller 与 Laurenczy 合作研究 将甲酸脱氢和二氧化碳加氢反应应用于可充电氢气电 池, 在一个连续产氢装置中, 基于 $\left[\mathrm{RuCl}_{2}\left(\mathrm{C}_{6} \mathrm{H}_{6}\right)\right]_{2} / \mathrm{DPPE}$ 催化剂, 以 $\mathrm{N}, \mathrm{N}$-二甲基己胺为添加剂, 在 $80{ }^{\circ} \mathrm{C}$ 和 101 $\mathrm{kPa}$ 下 TOF 达到 $47970 \mathrm{~h}^{-1[20]}$. 他们也解释了在三乙胺 存在等容条件下, 产生的氢气压力决定了热力学平衡, 从而决定了甲酸的转化率 ${ }^{[21]}$. 此后, Beller 等 ${ }^{[22]}$ 对几种 胺的篎选使系统的稳定性得到了改善, 在 $25{ }^{\circ} \mathrm{C}$ 和 $N, N$ 二甲基己胺存在下, $45 \mathrm{~d}$ TON 达到 1000000. 重要的是, 产生的气体混合物中只检测到 $\mathrm{H}_{2}$ 和 $\mathrm{CO}_{2}$, 未检测到 $\mathrm{CO}$, 这使得催化体系可以应用于燃料电池之中.

2004 年, Geldbach、Dyson 和 Ohta 等 ${ }^{[23-24]}$ 已经开始 研究离子液体(IL)作为溶剂和辅助催化剂在以 $\mathrm{FA} / \mathrm{NEt}_{3}$ 混合物中的转移加氢反应. 然而直到 2010 年, Shi 和 Deng 团队 ${ }^{[25]}$ 报道了使用一种胺功能化离子液体(IL1)作 为碱, 以 $\left[\mathrm{RuCl}_{2}(p \text {-cymene })\right]_{2}$ 为催化剂前体进行分解甲 酸产氢. 通过使用 IL, 可避免挥发性溶剂/添加剂的排 放及气体产物污染问题. 将甲酸钠和 ${ }^{i} \mathrm{Pr}_{2} \mathrm{NE}-\mathrm{MimCl}$ (EMim $=1$-乙基-3-甲基咪㛗离子)结合, IL 的量增加到

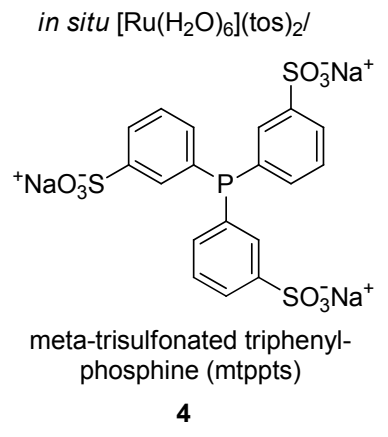

in situ $\left[\mathrm{RuCl}_{2} \text { (benzene) }\right]_{2} /$

图 3 Laurenczy 和 Beller 报道的催化体系

Figure 3 Catalytic system reported by Laurenczy and Beller

$10 \mathrm{mmol}$ ，同时减少催化剂的负载, TON 最大可达 1267 , 该体系的主要局限在于 FA 转化不完全, 以及在第一次 运行后催化剂失活. 2010 年, Dupont 等 ${ }^{[26]}$ 使用钉催化剂 $\left(\left[\mathrm{RuCl}_{2}(p \text {-cymene })\right]_{2}\right)$ 和离子液体 $\mathrm{Et}_{2} \mathrm{NEM}$-imCl (IL2)对 甲酸进行脱氢. 在 $80{ }^{\circ} \mathrm{C}$ 下 $7 \mathrm{~h}$ 内超过 $90 \%$ 的甲酸发生 脱氢, 初始 TOF 为 $1540 \mathrm{~h}^{-1}$. 该催化体系相对稳定, 而 且催化剂可以回收使用, 催化活性只有轻微的下降. 2011 年, Wasserscheid 等 ${ }^{[27]}$ 测试了 $\mathrm{RuCl}_{3}$ 和离子液体 IL3, 用于从 $\mathrm{FA} / \mathrm{NaOOCH}$ 生成 $\mathrm{H}_{2}$. [EMMim][OAc] (EMMim $=1$-乙基-2,3-二甲基咪唑离子)的催化活性最佳, 在 $80{ }^{\circ} \mathrm{C}$ 下反应 $84 \mathrm{~h}$, 甲酸转化率为 $91 \%$, 然而 [EMim][OAc]的催化活性只能达到 59\%. 在第一个循环中观察 到一个诱导期, 在 $80{ }^{\circ} \mathrm{C}$ 时随后的循环产生了更高且更 加稳定的 TOF $\left(150 \mathrm{~h}^{-1}\right)$. 作者提出诱导期与 $\mathrm{Ru}(\mathrm{III})$ 活性 物质还原为 $\mathrm{Ru}(\mathrm{II})$ 活性物质有关, 这一点通过对 $\mathrm{Ru}(\mathrm{II})$ 前体的进一步研究得到了证实 ${ }^{[28]}$.
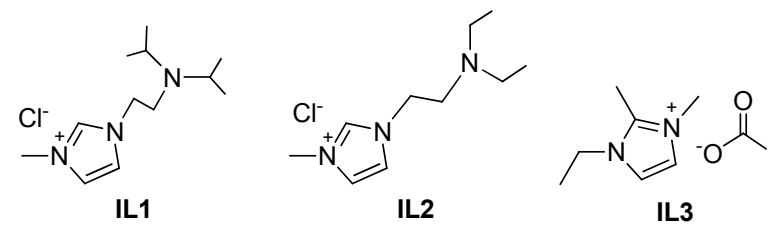

图 4 离子液体作为共催化剂体系

Figure 4 Ionic liquid as co-catalyst system

2011 年, Prakash 和 Olah 等 ${ }^{[29]}$ 研究了在 $109{ }^{\circ} \mathrm{C}$, 以 $\mathrm{RuCl}_{3}$ 为催化剂前体, 不含任何添加剂的 $\mathrm{FA} /$ $\mathrm{NaOOCH}$ 水溶液体系的甲酸脱氢反应. 该条件下，发生 了 FA 脱羧 $(0.21 \% \mathrm{CO})$. 在没有甲酸钠的情况下，反应 速率明显降低. 添加碱后使最初的活性得以恢复, 并分 离表征了一个四核 $\left[\mathrm{Ru}_{4}\left(\mathrm{HCO}_{2}\right)_{4}(\mathrm{CO})_{8}\left(\mathrm{PPh}_{3}\right)_{2}\right]$ 络合物 $(\boldsymbol{6})$, 且该络合物一旦形成, 就不容易发生 CO 解离. 他们还 研究了同一催化剂前体 $\mathrm{RuCl}_{3}$ 与许多稳定的膦配体的 $\mathrm{FA}$ 脱氢反应 ${ }^{[30]}$. 在循环过程中, 使用 $\mathrm{PPh}_{3} \cdot \mathrm{HBr}$ 后活性 有所改善, 这是因为它在水溶剂中的溶解度更高. 此外, 
从反应中得到了 $\left[\mathrm{Ru}\left(\mathrm{HCO}_{2}\right)_{2}(\mathrm{CO})_{2}\left(\mathrm{PPh}_{3}\right)_{2}\right] 、\left[\mathrm{Ru}(\mathrm{CO})_{3^{-}}\right.$ $\left.\left(\mathrm{PPh}_{3}\right)_{2}\right] 、\left[\mathrm{Ru}_{2}\left(\mathrm{HCO}_{2}\right)_{2}(\mathrm{CO})_{4}\left(\mathrm{PPh}_{3}\right)_{2}\right]$ 和一个 $\mathrm{Ru}_{12}$ 簇, 但相 对于原位形成的催化剂, 这些化合物对整体性能的贡献 有限. 2015 年, 作者研究了从甲酸盐中产生 $\mathrm{H}_{2}$ 的过程, 这使得 $\mathrm{H}_{2}$ 可以可逆地储存在碳酸氢盐中, 从而避免需 要额外的碱 ${ }^{[31]}$. 报道的两种脂肪族钓螯合催化剂 $(7,8)$, 1,4-二氧烷和水的混合物在 $69{ }^{\circ} \mathrm{C}$ 下, 初始 TOF 为 286 $\mathrm{h}^{-1}$ 时, 甲酸钠 $100 \%$ 脱氢. 另一种含有甲基的钓催化剂, 其初始 TOF 为 $430 \mathrm{~h}^{-1}$.
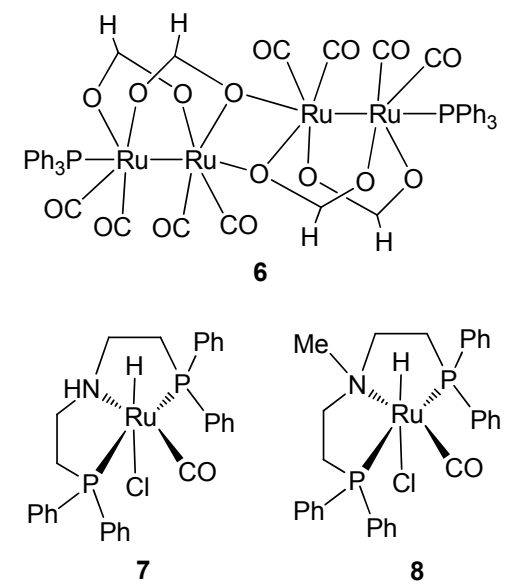

图 5 四核 $\left[\mathrm{Ru}_{4}(\mathrm{CO})_{12} \mathrm{H}_{4}\right]$ 络合物和脂肪族钉螯合物

Figure 5 Tetranuclear $\left[\mathrm{Ru}_{4}(\mathrm{CO})_{12} \mathrm{H}_{4}\right]$ complex and aliphatic ruthenium chelate

最近，一些带有钳型配体的金属化合物在选择性 FA 脱氢反应中表现出了很高的活性. 在 Nozaki 和 Milstein 等 ${ }^{[32-33]}$ 报道钳型 $\mathrm{Fe}$ 和 Re 催化剂之后, Pidko 和 同事 ${ }^{[34]}$ 研究了可逆 $\mathrm{H}_{2}$ 固定框架下钌 PNP 型螯合催化剂 的 FA 脱氢反应. 其中一种钟 PNP [2,6-双 (二叔丁基膦甲 基)-吡啶]鳌合物 (9), 当存在 DMF 和三乙胺时, 在 $90{ }^{\circ} \mathrm{C}$ 无 CO 污染的情况下, TOF 为 $256000 \mathrm{~h}^{-1}$, TON 超 过 706500. 作者还证明了反应决速步骤取决于碱的性 质: 对于较弱的 $\mathrm{NEt}_{3}$, 通过结合金属氢化物和质子化的 碱形成 $\mathrm{H}_{2}$, 要比 $\mathrm{C}-\mathrm{H}$ 键裂解更容易, 使得后者成为决 速步. 然而, 当存在更强的 1,5-二氮杂二环 [5.4.0]十一 烯-5 (DBU)时, 该速率被认为依赖于 $\mathrm{H}_{2}$ 生成步骤. 2016 年, Zheng 和 Huang 团队 ${ }^{[35]}$ 研究了三个含有去芳构化吡 啶和亚胺的手臂的 $\mathrm{PN}^{3}-\mathrm{Ru}$ 螯合物催化 $\mathrm{FA}$ 脱氢反应 $(10 \sim 12)$. 络合物在二甲基亚砜(DMSO)溶液活性最高, TON 和 TOF 分别为 95000 和 $2380 \mathrm{~h}^{-1}$ ，而在甲苯、乙腈、 DMF、四氢呋喃(THF)溶剂中催化剂的稳定性降低. 三 乙胺的引入和加热至 $90{ }^{\circ} \mathrm{C}$, 反应速率提高了 3 倍 $\left(\mathrm{TOF}=7333 \mathrm{~h}^{-1}\right)$, 稳定性也有所增加 $(\mathrm{TON}=1100000)$. 催化转化机制包括三个步骤: 将脱芳基螯合物的亚胺臂 质子化得到氢化物中间体, 甲酸盐分解并释放 $\mathrm{CO}_{2}$, 消 除氢以再生催化剂.

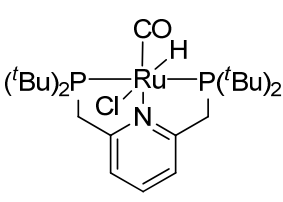

9

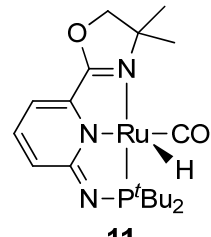

11

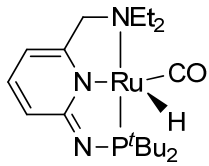

10

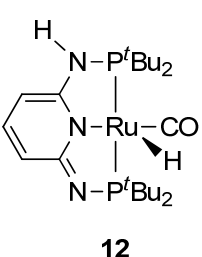

12
图 $6 \mathrm{PN}^{3}-\mathrm{Ru}$ 螯合物

Figure $6 \mathrm{PN}^{3}-\mathrm{Ru}$ chelate

\section{2 铱金属络合物}

1967 年 Coffey ${ }^{[2]}$ 发表了第一篇关于甲酸脱氢均相催 化剂的报告, 研究了带有膦配体的过渡金属络合物催化 甲酸分解的过程. 其中, $\left[\mathrm{IrH}_{3}\left(\mathrm{PPh}_{3}\right)_{3}\right]$ 使得甲酸的初始脱 氢率高达 $80 \mathrm{~mol} \cdot \mathrm{L}^{-1} \cdot \mathrm{h}^{-1}\left(\mathrm{TOF} \approx 8900 \mathrm{~h}^{-1}\right)$. 在随后的几 十年里, 人们研究了以铑、铱和钉为基础的催化剂, 但 它们的甲酸脱氢活性并不高.

在 2008 年报道了一种亲水性半三明治铑络合物之 后, 2010 年, Fukuzumi 等 ${ }^{[36]}$ 采用 $\left[\mathrm{Ru}^{\mathrm{II}}(\mathrm{bpy})_{2}(\mathrm{bpm})\right]\left(\mathrm{SO}_{4}\right)$ 与 $\left[\mathrm{Ir}^{\mathrm{III}}\left(\mathrm{Cp}^{*}\right)\left(\mathrm{H}_{2} \mathrm{O}\right)_{3}\right]\left(\mathrm{SO}_{4}\right)$ 在 $\mathrm{H}_{2} \mathrm{O}$ 中 $298 \mathrm{~K}$ 反应, 合成了 一种异二核铱钉络合物 $\left[\mathrm{Cp} * \mathrm{Ir}\left(\mathrm{H}_{2} \mathrm{O}\right)(\mathrm{bpm}) \mathrm{Ru}(\mathrm{bpy})_{2}\right]-$ $\left(\mathrm{SO}_{4}\right)_{2}(\mathrm{bpm}=2,2$ '-联嘧啶, bpy=联吡啶 $)(13)$, 该络合物 在 $25{ }^{\circ} \mathrm{C}$ 时甲酸钠存在的状况下, 催化甲酸选择性脱氢. $\mathrm{TOF}$ 随着 $\mathrm{HCOOH}$ 与 $\mathrm{HCOONa}$ 混合溶液中甲酸盐总浓 度的增加而逐渐增大, 在 $\mathrm{pH}=3.8$ 时 $\mathrm{TOF}$ 达到最大为 $426 \mathrm{~h}^{-1}$. 由[ $\left.\mathrm{HCOO}^{-}\right]$对 TOF 的影响表明, $\mathrm{H}_{2}$ 的生成经过 $\left[\mathrm{II}^{\mathrm{III}}\left(\mathrm{Cp}^{*}\right)(\mathrm{O}(\mathrm{CO}) \mathrm{H})(\mathrm{bpm}) \mathrm{Ru}^{\mathrm{II}}(\mathrm{bpy})_{2}\right]^{3+}$ 这种复杂的络合 物. 并且当 $\mathrm{pH}>3.8$ 时 TOF 降低, 表明反应的决速步骤 是氢化物络合物 $\left[\mathrm{Ir}^{\mathrm{III}}\left(\mathrm{Cp}^{*}\right)(\mathrm{H})(\mathrm{bpm}) \mathrm{Ru}^{\mathrm{III}}(\mathrm{bpy})_{2}\right]^{3+}$ 的反应. 2012 年, Fukuzumi 等 ${ }^{[37]}$ 以三水络合物 $\left[\operatorname{Ir}^{\mathrm{III}}\left(\mathrm{Cp}^{*}\right)\right.$ $\left.\left(\mathrm{H}_{2} \mathrm{O}\right)\right] \mathrm{SO}_{4}$ 与 4-(1 $\mathrm{H}$-吡唑-1-酰基)苯甲酸在水中回流反应 合成了水溶性铱水络合物(14), 用于催化分解甲酸产生 氢气，在 $298 \mathrm{~K}$ 和 $\mathrm{pH}=2.8$ 时, TOF 最大为 $1880 \mathrm{~h}^{-1}$, 随 着 $\mathrm{pH}$ 的升高, 络合物发生去质子反应, TOF 也随之减 少, 当 $\mathrm{pH}=9.0$ 时, $\mathrm{TOF}$ 为 0 . 此外, 该络合物在常温低 压的碱性水中对碳酸氢盐加氢反应也具有较高的催化 活性.

Himeda ${ }^{[38]}$ 自 2009 年报道了甲酸脱氢反应催化剂以 来，篮选并优化了大量的水溶性金属络合物和及其反应 条件，显著改善催化剂的效率和稳定性，并详细深入研 究反应机理. 他们最初研究了一系列含有双吡啶基的 铱、铑和钉金属络合物 $\mathbf{1 5} \sim \mathbf{2 0}$, 其中 [Cp* $\operatorname{Ir}\left(4,4^{\prime}-\mathrm{dhbp}\right)-$ $\left.\left(\mathrm{H}_{2} \mathrm{O}\right)\right] \mathrm{SO}_{4}\left(\mathbf{1 5}, \mathrm{dhbp}=4,4^{\prime}\right.$-二羟基-2,2'-联吡啶)在 $90{ }^{\circ} \mathrm{C}$ 


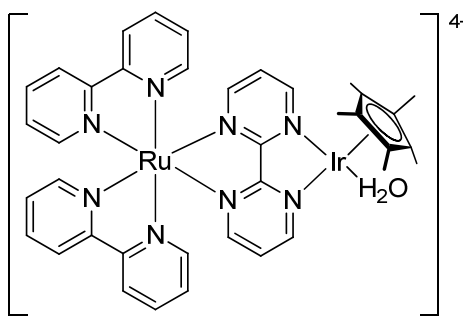

13

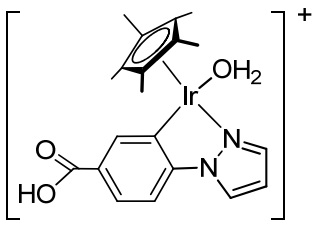

14
图 7 Fukuzumi 报道的异二核铱钉络合物和铱水络合物

Figure 7 Heterodinuclear iridium ruthenium complex and iridium water complex reported by Fukuzumi

的 $2 \mathrm{~mol} \cdot \mathrm{L}^{-1}$ 甲酸水溶液中的 TOF 达到 $14000 \mathrm{~h}^{-1}$, 由于 吡啶配体的羟基取代基的两种形式之间存在酸碱平衡, 因此对溶液 $\mathrm{pH}$ 有很强的依赖性. 在 $60{ }^{\circ} \mathrm{C}$ 时, 没有甲酸 盐的情况下, 当 $\mathrm{pH}=2$ 时 $\mathrm{FA}$ 水溶液中初始的 TOF 达到 $2500 \mathrm{~h}^{-1}$, 而当 $\mathrm{pH}>4.5$ 时催化剂几乎没有活性. 在 4 $\mathrm{mol} \cdot \mathrm{L}^{-1}$ 甲酸溶液中, 初始 $\mathrm{TOF}$ 最高可达到 $3000 \mathrm{~h}^{-1}$ 以 上, 但随着甲酸浓度进一步增加 TOF 反而降低. 研究表 明铱催化剂的 TOF 值与 Hammett 取代基常数有较好的 相关性, 即具有较强供电子的双吡啶配体有较高的反应 速率. 2012 年, Himeda 与其他研究小组 ${ }^{[39]}$ 合作开发了一 种可对 $\mathrm{pH}$ 响应的铱络合物 $\mathbf{2 1} \sim \mathbf{2 3}$, 在 $90{ }^{\circ} \mathrm{C}$ 条件下催 化 FA 脱氢, 其中 22 的初始 TOF 为 $228000 \mathrm{~h}^{-1}$. 虽然甲 酸和甲酸盐都可作为底物, 但后者的脱氢效率明显较 低. 2014 年, 他们 ${ }^{[40]}$ 报道了 $\left(\mathrm{Cp} * \operatorname{Ir}(\right.$ thbpm $\left.)\left(\mathrm{H}_{2} \mathrm{O}\right)\right) \mathrm{SO}_{4}$ (thbpm $=2,2^{\prime}, 6,6^{\prime}$-四羟基-4,4'-联嘧啶)络合物(24), 在 1 $\mathrm{mol} \cdot \mathrm{L}^{-1}$ 的 $\mathrm{FA} / \mathrm{NaOOCH}$ 溶液中, 初始 TOF 达到 39500 $\mathrm{h}^{-1}$. 在相同条件且没有甲酸钠情况下, 另一种铱催化 剂 $\left[\mathrm{Cp} * \operatorname{Ir}(\mathrm{tmbi})\left(\mathrm{H}_{2} \mathrm{O}\right)\right] \mathrm{SO}_{4}$ (tmbi=四甲基二咪唑) (25)表

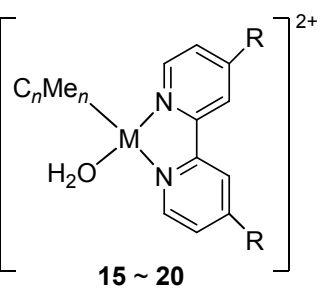

15: $n=5 ; \mathrm{M}=\mathrm{Ir} ; \mathrm{R}=\mathrm{OH}$ 16: $n=5 ; \mathrm{M}=\mathrm{Rh} ; \mathrm{R}=\mathrm{OH}$ 17: $n=6 ; \mathrm{M}=\mathrm{Ru} ; \mathrm{R}=\mathrm{OH}$ 18: $n=5 ; \mathrm{M}=\mathrm{Ir} ; \mathrm{R}=\mathrm{OMe}$ 19: $n=5 ; \mathrm{M}=\mathrm{Ir} ; \mathrm{R}=\mathrm{Me}$ 20: $n=5 ; \mathrm{M}=\mathrm{Ir} ; \mathrm{R}=\mathrm{H}$

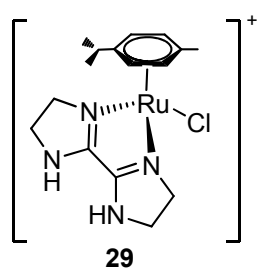

现出相当的活性 $\left(\mathrm{TOF}=34000 \mathrm{~h}^{-1}\right)^{[41]} .2015$ 年，作者 ${ }^{[42]}$ 提出了含有功能化的嘧啶基咪唑啉配体的铱催化剂 (26), 在甲酸盐存在下, $100{ }^{\circ} \mathrm{C}$ 时的 TOF 可达 322000 $\mathrm{h}^{-1}$. 此外, 他们演示了一个潜在的 FA 脱氢装置, 可以 产生 $1.02 \mathrm{~m}^{3}$ 的 $\mathrm{H}_{2}\left(p_{\max }=1.0 \mathrm{MPa}\right)$, 采用吡啶基咪唑啉 基铱络合物(27) ${ }^{[43]}$, 在 $363 \mathrm{~h}$ 后的 TON 为 2000000 . 该催 化剂在酸性环境 $(\mathrm{pH}=1.7)$ 中十分稳定, 在不需要碱的 情况下, 平均 $\mathrm{TOF}$ 为 $6250 \mathrm{~h}^{-1}$. 随后, Himeda 和 Kawanami 等 ${ }^{[44]}$ 研究了一种含有 1,10 -邻二氮菲- 4,7 -二醇 配体的铱络合物(28), 在 $60{ }^{\circ} \mathrm{C}$ 时催化剂保持稳定性和 活性长达 3.5 个月, 甲酸 $\left(10 \mathrm{~mol} \cdot \mathrm{L}^{-1}\right) 100 \%$ 分解产生 $\mathrm{H}_{2}$ 且不含 $\mathrm{CO}, T O N$ 为 $5000000\left(\mathrm{TOF}=3010 \mathrm{~h}^{-1}\right)$. 为了寻 求更高效的催化剂, Himeda 和 Huang 等 ${ }^{[45]}$ 开发了一种含 有 2,2-联吡唑配体的钉络合物 (29). $\mathrm{NaOOCH} / \mathrm{HCOOH}$ 的最佳比例为 $5: 1$, 在 $90{ }^{\circ} \mathrm{C}$ 条件下反应前 $10 \mathrm{~min}$ 内, TOF 最高可达 $12000 \mathrm{~h}^{-1}$. 在没有碱的情况下，催化剂初 始 TOF 降低为 $3750 \mathrm{~h}^{-1}$. 在催化效率实验中, $35 \mathrm{~h}$ 后催 化剂的 TON 达到 350000. 最近, Himeda 等 ${ }^{[46]}$ 报道了 $\left[\mathrm{Cp}^{*} \operatorname{Ir}\left(4,4^{\prime}-\mathrm{dhbp}\right)\left(\mathrm{H}_{2} \mathrm{O}\right)\right] \mathrm{SO}_{4}$ 催化剂用于选择性 FA 脱氢, 在 $\mathrm{H}_{2} / \mathrm{CO}_{2}$ 压力为 $120.0 \mathrm{MPa}$ 和 $80{ }^{\circ} \mathrm{C}$ 反应条件下, 生成 的气体混合物存在超临界相, 因此可以通过简单的降低 温度来净化 $\mathrm{H}_{2}$. 作者认为以这种方式从 $\mathrm{FA}$ 中释放 $\mathrm{H}_{2}$ 可以充分满足燃料电池汽车的需要.

2013 年, Xiao 和 Barnard 等 ${ }^{[47]}$ 研究了 $25{ }^{\circ} \mathrm{C}$ 共沸三 乙胺甲酸 $\left(5 \mathrm{FA} / 2 \mathrm{NEt}_{3}\right)$ 体系, 考察了环化铱催化剂各组 成部分对其 FA 脱氢活性的影响(图 9). 一方面研究了 $\mathrm{N}$ 供体组的影响，即带有 $\mathrm{N}-\mathrm{H}$ 质子的亚胺基络合物 $(\mathbf{L 1} \sim \mathbf{L 5})$ 的初始 TOF 在 500 1000 $\mathrm{h}^{-1}$, 而 $\mathrm{N}$-杂环 $(\mathbf{L 6}$,

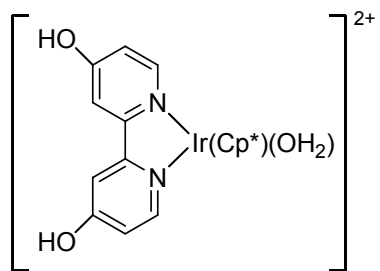

21

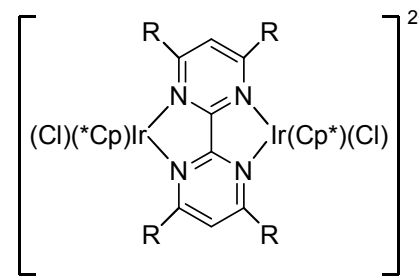

22: $\mathrm{R}=\mathrm{OH} ; 23: \mathrm{R}=\mathrm{H}$

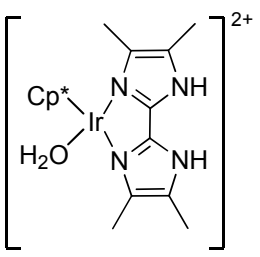

25

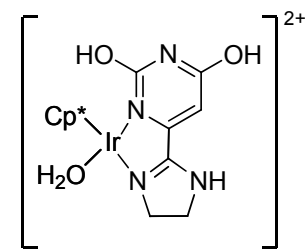

26

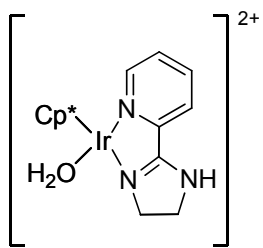

27

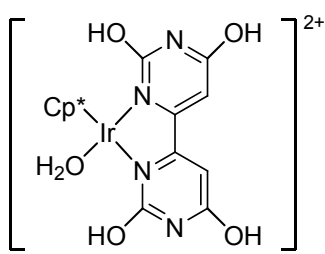

24
图 8 Himeda 等报道的金属络合物催化剂

Figure 8 Metal complex catalysts reported by Himeda et al. 
L7)、亚胺 $(\mathrm{LB}, \mathrm{L9})$ 和饱和苄胺 $(\mathrm{L10})$ 的络合物活性不高. 当 $\mathrm{N}-\mathrm{H}$ 质子被供电子 $(\mathbf{L 1 1}, \mathbf{L 1 2})$ 或吸电子 $(\mathbf{L 1 3})$ 取代, 导致催化剂失活, 说明 $\mathrm{N}-\mathrm{H}$ 质子对于催化起到关键作 用. 通过在配体上引入苯基(L4, L5), 可以提高催化剂 的使用寿命, 从而克服在空间要求较低的情况下催化剂 快速失活的问题 $(\mathbf{L 1} \sim \mathbf{L 3})$. 另一方面, 研究了环化基团 (L14 L18) 对甲酸脱氢的影响, 发现具有强电子释放取 代基的活性更高, 其中以 $\mathbf{L 1 8}$ 为配体的络合物的最大初 始 TOF 为 $1960 \mathrm{~h}^{-1}$. 作者还发现咪唑啉环 $\mathrm{C} 2$ 位置进行 环化反应可得到最优构型, 并使用优化的铱催化剂 [Cp* $\mathrm{IrCl}(\mathbf{L 1 9})]$, 在 $25{ }^{\circ} \mathrm{C}$ 初始 TOF 最高可达到 2570 $\mathrm{h}^{-1}$. 由于反应速率与 $\mathrm{FA}$ 浓度成正比, 因此在 $40{ }^{\circ} \mathrm{C}$ 连 续制氢装置中, 加入足量的 FA, 反应速率急剧增加至 $147000 \mathrm{~h}^{-1}$, 持续时间为 $10 \mathrm{~s}$.

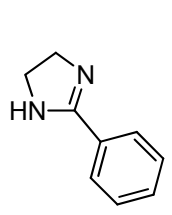

L1
L2

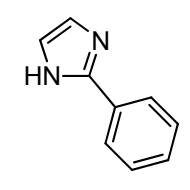<smiles>c1ccc(C2=N[C@@H]3CCCC[C@H]3N2)cc1</smiles>

L3

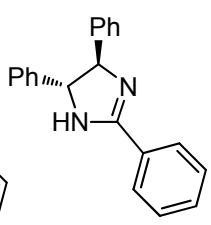

L4<smiles>COc1ccc(C2=N[C@H](c3ccccc3)[C@H](c3ccccc3)N2)cc1</smiles><smiles>c1ccc(C2=NCCO2)cc1</smiles>

L7
L5<smiles>COc1ccc(/N=C(\C)c2ccc(OC)cc2)cc1</smiles>

L9<smiles>c1ccc(CN2CCN=C2c2ccccc2)cc1</smiles>

L12 L6 $\mathrm{MeO}$<smiles>CC(Nc1ccc(O)cc1)c1ccccc1</smiles><smiles>CN1CCN=C1c1ccccc1</smiles>

L11

L18

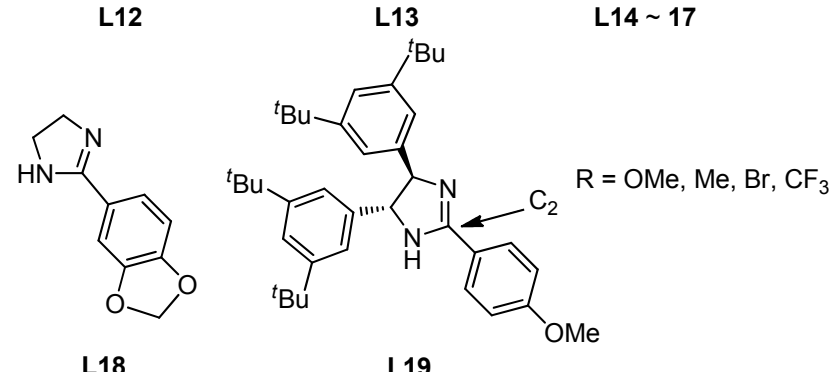<smiles>CC(=O)N1CCN=C1c1ccccc1</smiles>

L13

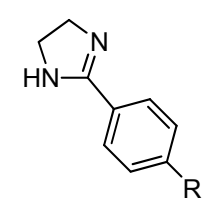

L14 17

图 9 Xiao 等报道的环化铱催化剂的配体

Figure 9 Ligands for cyclized iridium catalysts reported by Xiao et al.

2011 年, Nozaki 等 ${ }^{[48]}$ 采用铱 PNP ${ }^{1}$ 螯合物三氢化物 络合物(30)对 FA 进行脱氢反应. 该催化剂在 FA/TEA 和 叔丁醇混合物中, $80{ }^{\circ} \mathrm{C}$ 时反应 $1 \mathrm{~min}$ 后的 TOF 可达到 $120000 \mathrm{~h}^{-1}$, 在 $4 \mathrm{~h}$ 后下降到 $1200 \mathrm{~h}^{-1}$, 甲酸完全分解的 TON 值为 5000. 2017 年, Fink 和 Laurenczy ${ }^{[49]}$ 报道了一 系列 $\mathrm{Rh}(\mathrm{III})$ 和 $\mathrm{Ir}(\mathrm{III})$ 基催化剂下甲酸 $\left(4 \mathrm{~mol} \cdot \mathrm{L}^{-1}\right)$ 脱氢, 具有多种电子和空间性质的双齿二氮配体使得催化剂 的稳定性提高, 但它们的活性一般, 在 $\left[\mathrm{Cp}^{*} \operatorname{Ir}\left(\mathrm{C}_{6} \mathrm{H}_{14^{-}}\right.\right.$ $\mathrm{N}_{2}$ ) Cl] Cl 的存在下, $90{ }^{\circ} \mathrm{C}$ 时最大 TOF 为 $3278 \mathrm{~h}^{-1} .2019$ 年, Onishi 等 ${ }^{[50]}$ 开发出了含有吡啶和不同的吡唑基 $N, N$ 二齿配体的 $\mathrm{Cp} * \mathrm{Ir}$ 络合物 $(\mathbf{3 1} \sim \mathbf{3 4})$ 用于 $\mathrm{FA}$ 脱氢. 在吡唑 环上引入 $\mathrm{OH}$ 基团提高了催化活性，这一趋势与吡啶型 配体相似. 该催化剂在甲酸脱氢过程中保持了 $35 \mathrm{~d}$ 的催 化活性, 在 $4 \mathrm{~mol} \cdot \mathrm{L}^{-1}$ 的甲酸中进行脱氢反应, TON 可达 到 100000 .

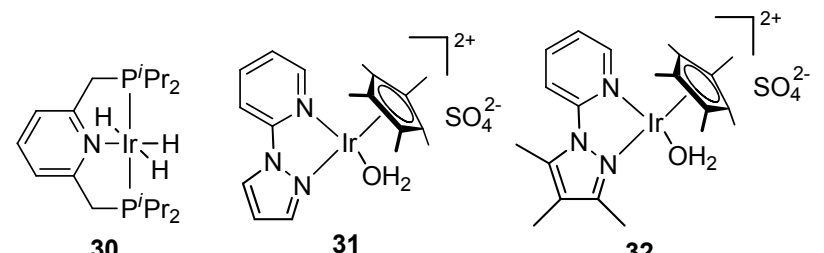

30

31
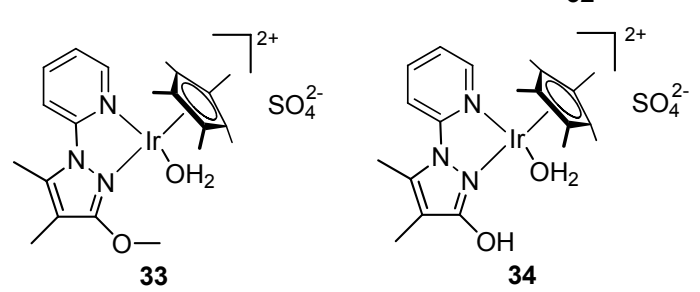

图 10 Nozaki 和 Onishi 等报道的铱催化剂

Figure 10 Iridium catalysts reported by Nozaki and Onishi et al.

2018 年, 李灿等 ${ }^{[51]}$ 研发了一种新型二氨基乙二醛 肜衍生铱催化剂体系(35 37), 该体系在没有添加剂的 情况下，通过甲酸在水中的分解，实现了高效产氢. 这 些催化剂由 $\left[\mathrm{IrCp}^{*} \mathrm{Cl}_{2}\right]_{2}$ 和二肟配体原位制备得到. 胺取 代基二肟配体具有更多电子，使催化剂体系具有较高的 稳定性. 并且可以根据实际需要，在高温或低温下都能 高效工作，在 $90{ }^{\circ} \mathrm{C}$ 时 TON 达到 3900000(平均速率 $\mathrm{TOF}=65000 \mathrm{~h}^{-1}$ ), 在 $70{ }^{\circ} \mathrm{C}$ 时 $\mathrm{TON}$ 达到 5020000(平均 速率 $\mathrm{TOF}=28700 \mathrm{~h}^{-1}$ ), 在 $25 \sim 26{ }^{\circ} \mathrm{C}$ 时 $\mathrm{TON}$ 达到 400000(平均速率 $\mathrm{TOF}=1053 \mathrm{~h}^{-1}$ ). 该催化剂具有制备 简单、效率高、长期稳定等优点，使其更适合于甲酸脱 氢.

\section{3 铑金属络合物}

2008 年, Fukuzumi 等 ${ }^{[52]}$ 报道了一种亲水性的半三 明治结构的铑络合物 $\left[\mathrm{Cp} * \mathrm{Rh}\left(\mathrm{H}_{2} \mathrm{O}\right)(\mathrm{bpy})\right]^{2+}(\mathrm{bpy}=$ 联吡 

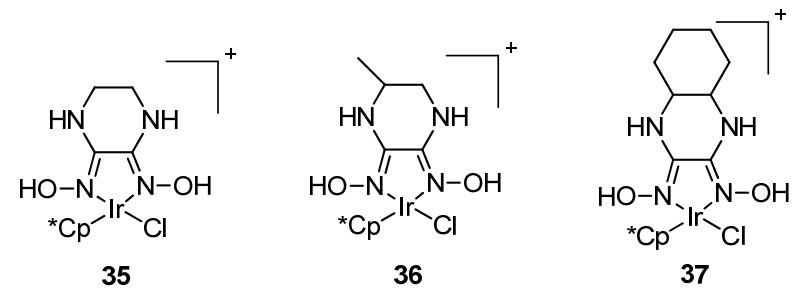

图 11 二氨基乙二醛肟衍生铱催化剂体系

Figure 11 Diaminoglydoxime derived iridium catalyst system

啶)(38), 该络合物在 $25{ }^{\circ} \mathrm{C}$ 时甲酸钠存在下的情况下, 对 于甲酸的选择性脱氢具有催化活性. 当 $\mathrm{pH}=3.8$ 时, 刚 好与甲酸的 $\mathrm{p} K_{\mathrm{a}}$ 值相对应，最高 TOF 可达到 $28 \mathrm{~h}^{-1}$. 而 当 $\mathrm{pH}$ 大于 $\mathrm{p} K_{\mathrm{a}}$ 值时, 由于形成了非活性的羟基络合物 $[\mathrm{Cp} * \mathrm{Rh}(\mathrm{OH})(\mathrm{bpy})]^{+}$, 导致催化剂失活.

2016 年, 李灿等 ${ }^{[53]}$ 报道了催化剂 $\left[\left(\mathrm{Cp}^{*} \mathrm{RhCl}_{2}\right)\right]_{2}$ 二聚 物(39)在共沸 $\mathrm{FA} / \mathrm{NEt}_{3}$ 中的脱氢反应. 当添加卤素阴离 子, 尤其是碘离子的加入使得催化剂的活性和稳定性显 著提高 $\left(60{ }^{\circ} \mathrm{C}\right.$ 时, TON (30 min) 为 $\left.706, \mathrm{TOF}=4375 \mathrm{~h}^{-1}\right)$. 值得注意的是, 该体系并不需要添加稳定的配体. 研究 表明, 卤化物影响了决速步骤, 活性催化剂是单核的, 在催化循环外与双核氢化物处于平衡状态. TOF 值在较 高的 FA 比时下降, 直到在纯底物中降为 0 , 说明氢化物 的形成限制了脱氢反应. 此外, 叔胺比伯胺和仲胺更合 适作为反应的添加物, 主要是因为后两者和碘离子之间 的氢键更强, 从而降低了溶液中卤化物的有效性. 由于 碘化物对金属的亲和力比氯化物更强, 促进甲酸盐络合 物通过释放二氧化碳生成相应氢化物.

2016 年, Reek 等 ${ }^{[54]}$ 报道了 $\mathrm{Rh}(\mathrm{CO})(\mathrm{L})[\mathrm{L}=2$-二(叔丁 基膦甲基)-6-苯基吡啶] (40)用于无碱 FA 脱氢, 其中金 属-碳部分起到碱的作用. 在 $75{ }^{\circ} \mathrm{C}$ 条件下, 将 1,4-二恶 烷中加入 $0.5 \mathrm{~mol} \%$ 铑进行催化反应, 在连续 8 次运行中, TOF 稳定为 $169 \mathrm{~h}^{-1}$, 而三乙胺并没有起到增强催化活 性的作用. 研究表明可逆的环金属化反应可以促进甲酸 活化, 在添加 $\mathrm{FA}$ 时, $\mathrm{Rh}-\mathrm{C}$ 键断裂产生金属-甲酸盐 络合物, 苯基连接的起始络合物在底物被完全消耗后重 新形成. 作者认为, 用氯、吡啶或 $\mathrm{P}\left({ }^{t} \mathrm{Bu}\right)_{2}$ 基取代苯环会 导致活性抑制, 这表明配体的配位数和金属上的配位点 对于催化活性至关重要.

2016 年, Kühn 等 ${ }^{[5]}$ 报道了一种具有双- $N$ 杂环碳烯 的铑络合物(41) 在水溶液和无添加剂条件下选择性催化 FA 脱氢. 该催化剂在 $25{ }^{\circ} \mathrm{C}$ 下的 $\mathrm{TOF}$ 已经达到了 90 $\mathrm{h}^{-1}$, 这是少有的性能优异的铑催化剂. 通过优化溶液 $\mathrm{pH}$ 值并逐渐降低催化剂用量, 在 $100{ }^{\circ} \mathrm{C}$ 时最大 $\mathrm{TOF}$ 为 $39200 \mathrm{~h}^{-1}$. 在此条件下, 催化剂稳定使用 $50 \mathrm{~h}$ 以上, TON 为 449000. 为进一步证明催化稳定性, 他们测试了 铑络合物在 $80{ }^{\circ} \mathrm{C}$ 下连续 $72 \mathrm{~h} \mathrm{FA}$ 脱氢反应, $\mathrm{TOF}$ 为 4300

$\mathrm{h}^{-1}$.

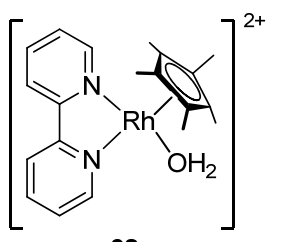

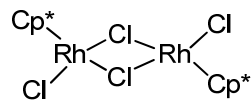

39

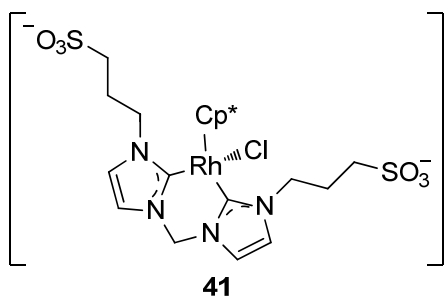

图 12 常见的铑催化剂

Figure 12 Common rhodium catalyst

\section{4 非贵金属催化剂}

尽管贵金属催化剂在选择性脱氢上取得了重要进 展，但用非贵金属替代贵金属也成为了科研工作者的研 究目标. 非贵金属催化剂主要优势在于其金属丰富性, 以及大规模应用情况下具有低成本等优点. 此外, 大自 然中有效的脱氢反应是由非贵金属活性位点 $(\mathrm{Fe}, \mathrm{Ni})$ 酶 催化的. 近年来, 人们已经开发了铁、钴、镍、铝等非 贵金属甲酸脱氢催化剂.

2010 年, Beller 等 ${ }^{[56]}$ 报道了几种非贵金属羰基化合 物 $\left[\mathrm{Fe}_{3}(\mathrm{CO})_{12}\right] 、\left[\mathrm{Mo}(\mathrm{CO})_{6}\right] 、\left[\mathrm{Mn}_{2}(\mathrm{CO})_{10}\right] 、\left[\mathrm{Cr}(\mathrm{CO})_{6}\right]$ 和 $\left[\mathrm{Co}(\mathrm{CO})_{8}\right]$ 等与不同膦和三联吡啶原位应用于 $\mathrm{FA}$ 溶液脱 氢. 其中, 由 $\left[\mathrm{Fe}_{3}(\mathrm{CO})_{12}\right] / \mathrm{PPh}_{3} / \mathrm{TPY}$ 生成的催化剂 42 稳 定性较差, 在 $60{ }^{\circ} \mathrm{C}$ 时的最大初始 TOF 为 $200 \mathrm{~h}^{-1}$. 通过 光谱和密度泛函理论(DFT)研究发现, 铁羰基/膦催化剂 只在光的存在下形成活性铁氢化物. 随后, 用 $\mathrm{PBn}_{3}$ 取代 $\mathrm{PPh}_{3}$ 形成了一种更稳定催化剂 $\mathbf{4 3}^{[57]}$, 在相同的反应条 件下, $\left[\mathrm{Fe}_{3}(\mathrm{CO})_{12}\right] / \mathrm{PBn}_{3} / \mathrm{TPY}$ 催化体系 $51 \mathrm{~h}$ 内 TON 达到 1266. 作者还指出催化剂的稳定性与邻位金属化之间存 在联系

2011 年, Beller 和 Laurenczy 合作 ${ }^{[58]}$ 开发了一种由 $\mathrm{Fe}\left(\mathrm{BF}_{4}\right)_{2} \cdot 6 \mathrm{H}_{2} \mathrm{O}$ 和 $\mathrm{PP}_{3}$ 在碳酸酯中组成的高活性催化体 系(44), 其 TOF 可达 $9425 \mathrm{~h}^{-1}$, 在 $80{ }^{\circ} \mathrm{C}$ T TON 超过 92000. 此外, 他们采用 $\mathrm{Fe}\left(\mathrm{BF}_{4}\right)_{2} \cdot 6 \mathrm{H}_{2} \mathrm{O}$ 或 $\left[\mathrm{FeH}\left(\mathrm{PP}_{3}\right)\right]-$ $\left(\mathrm{BF}_{4}\right)_{2}\left(\mathrm{BF}_{4}\right)_{2}$ 以及 2 倍 $\mathrm{PP}_{3}$, 在 $40{ }^{\circ} \mathrm{C}$ 下 $3 \mathrm{~h}$ 的 $\mathrm{TON}$ 约 1900 , 而 $\left[\mathrm{Co}\left(\mathrm{BF}_{4}\right)_{2} \cdot 6 \mathrm{H}_{2} \mathrm{O}\right]$ 和 $\left[\mathrm{Mn}(\mathrm{acac})_{2}\right]$ 几乎无活性. 其 中 $\mathrm{PP}_{3}$ 配体既作为碱也起到了稳定金属中心的作用 ${ }^{[59]}$. 最近, 将多齿 ${ }^{s} \mathrm{PP}_{3}$ 配体磺化原位生成铁(II)催化剂, TOF 在 $80{ }^{\circ} \mathrm{C}$ 下可达 $240 \mathrm{~h}^{-1}\left(\mathrm{FeCl}_{2}:{ }^{s} \mathrm{PP}_{3}, m: m=1: 2\right)$, 这 是在没有添加剂的水介质中起催化作用的铁催化剂 ${ }^{[60]}$.

2013 年, Milstein 等 ${ }^{[32]}$ 用铁 PNP $^{3}$ 螯合物 $\mathbf{4 5} \sim \mathbf{4 7}$ 在 $2 \mathrm{FA} / 1 \mathrm{NEt}_{3}$ 中选择性催化甲酸脱氢. 研究表明加入 $\mathrm{THF}$ 、 
1,4-二恶烷和 DMSO 可以使催化活性升高, 而在 EtOH、 $\mathrm{MeCN}$ 和水中活性降低. 在 $40{ }^{\circ} \mathrm{C}$ 时 $50 \mathrm{~mol} \%$ 胺存在下, TOF 为 $520 \mathrm{~h}^{-1}$. 在催化剂用量仅为 $0.001 \mathrm{~mol} \%$ 的情况 下, $10 \mathrm{~d}$ 内 TON 为 100000 . 此外, 在初始 $\mathrm{H}_{2} / \mathrm{CO}_{2}$ 压力为 $0 \sim 1.0 \mathrm{MPa}$ 时, 甲酸脱羧没有发生抑制作用, 说明在特 定的条件下, 反应是不可逆的.

2014 年, Hazari 和 Schneider 等 ${ }^{[61]}$ 研究表明铁 PNP ${ }^{2}$ 螯合物 48 49 在不需要碱的情况下, 以路易斯酸作为 辅催化剂, 1,4-二恶烷作为溶剂催化甲酸脱氢. 路易斯 酸对羧酸盐更高的亲和力使得活性增强, 这归因于结合 的甲酸盐中间体十分稳定. 当 $\mathrm{LiBF}_{4}$ 为 $10 \mathrm{~mol} \%$, 催化 剂浓度仅为 $0.0001 \mathrm{~mol} \%$, 在 $80{ }^{\circ} \mathrm{C}$ 下运行 4 次, TOF 大 于 $196000 \mathrm{~h}^{-1}$, TON 大于 980000 .

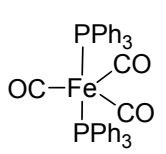

42

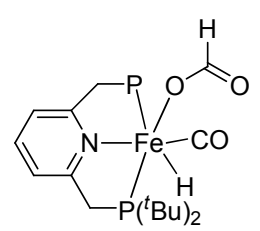

46

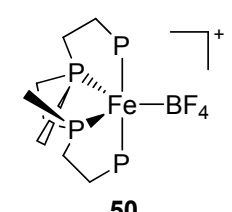

50

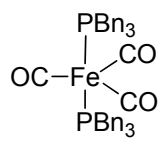

43

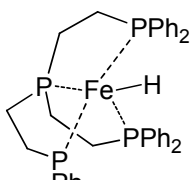

$\mathrm{Ph}_{2} 44$

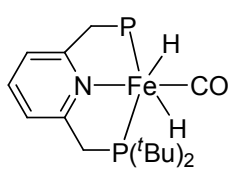

45

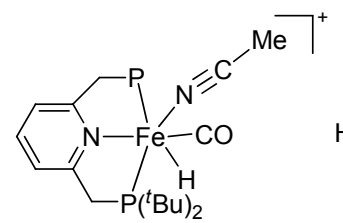

47

$\mathrm{Me}$

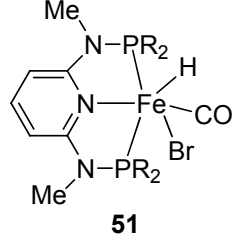

图 13 常见的铁催化剂

Figure 13 Common iron catalysts

2015 年, Gonsalvi 等 ${ }^{[62]}$ 以 $\left[\mathrm{Fe}(\mathrm{rac}-\mathrm{P} 4)\left(\mathrm{CH}_{3} \mathrm{CN}\right)_{2}\right]\left(\mathrm{BF}_{4}\right)$ ( $\mathrm{P} 4=1,1,4,7,10,10$-六苯基-1,4,7,10-四环磷酰胺膦) (50) 为催化剂, 在碳酸丙烯酯中进行甲酸无碱脱氢. 在 $40{ }^{\circ} \mathrm{C}$ 下, 催化剂形成 $\mathrm{Fe}\left(\mathrm{BF}_{4}\right)_{2} \cdot 6 \mathrm{H}_{2} \mathrm{O} / \mathrm{P} 4$ (meso/rac $=3$ ) 时, 反应 $6 \mathrm{~h}$ 后甲酸的转化率只有 $4 \%$. 当 $\mathrm{Fe}(\mathrm{II}) / \mathrm{rac}-\mathrm{P} 4$ $(m: m=1: 1)$ 时, $60 \%$ 的甲酸以 $35 \mathrm{~h}^{-1}$ 的初始速率脱氢. 当中心离子结合 2 个配体时甲酸完全转化, 其 TOF 可达 到 $139 \mathrm{~h}^{-1}$. 然而在 $40{ }^{\circ} \mathrm{C}$ 的循环试验表明, 进行到第三 个循环时, 催化活性已经下降了 $70 \%$. 当 $\mathrm{Fe}(\mathrm{II}) / \mathrm{rac}-\mathrm{P} 4$ (1：4)时, TON 由原来的 6061 增加到 10000. 此外, 无论 纯 meso-P4 配体与铁的比例如何, 催化活性都很低.

2016 年, Kirchner 和 Gonsalvi 等 ${ }^{[63]}$ 研究了碱、添加 剂、溶剂、温度和催化剂负载对 Fe-PNNNP ${ }^{1}$ 催化的 FA 脱氢的影响. 在无碱的情况下不发生反应, 而在 THF 中
加入 1 equiv. $\mathrm{NEt}_{3}$ 时, $5 \mathrm{~mol} \cdot \mathrm{L}^{-1} \mathrm{FA}$ 溶液完全反应，且反 应速率在 $610 \sim 720 \mathrm{~h}^{-1}\left(60{ }^{\circ} \mathrm{C}\right)$. 当路易斯酸取代碱时, 所有催化剂的 FA 脱氢反应被完全抑制. 此外, 在非质 子溶剂如 THF、PC 和 1,4-二恶烷中, 可以获得更高的反 应速率和总 FA 转化率. 在络合物 $\mathbf{5 1}$ 存在的情况下, 将 $\mathrm{FA}$ 浓度增加一倍至 $10 \mathrm{~mol} \cdot \mathrm{L}^{-1}$, 并将催化剂负载降低到 $0.01 \mathrm{~mol} \%$, 得到最高的 TOF $\left(2635 \mathrm{~h}^{-1}\right)$, 并在 $80{ }^{\circ} \mathrm{C}$ 条 件下 $6 \mathrm{~h}$ 后完全转换. 该催化剂可以重复利用, TON 高 于 12000 .

2014 年, Myers 和 Berben ${ }^{[64]}$ 首次研究了铝络合物 52 53 对甲酸脱氢反应的影响，提出了一种协同的金 属配体氢键参与碳氢键的裂解过程. 当 $\mathrm{FA} / \mathrm{NEt}_{3}(m$ : $m=5: 2$ )时, THF 作为溶剂, 加入 $0.006 \mathrm{~mol} \%$ 催化剂, 初始 TOF 可达 $5200 \mathrm{~h}^{-1}$. 甲酸脱氢可通过催化剂活化形 成双质子化的 $\left[\left({ }^{\mathrm{Ph}} \mathrm{H}_{2} \mathrm{I}_{2} \mathrm{P}\right) \mathrm{Al}(\mathrm{OOCH})_{2}\right]^{+}$络合物, 然后通过 $\beta$-氢化物消除释放 $\mathrm{CO}_{2}$ 和 $\mathrm{H}_{2}$, 并进一步使甲酸质子化.

2015 年, Enthaler 等 ${ }^{[65}$ 报道了 $[(\mathrm{PCP}) \mathrm{Ni}(\mathrm{H})]$ 催化剂 54. 在常温下反应时分离出中间产物 $[(\mathrm{PCP}) \mathrm{Ni}-$ $\left(\mathrm{H}_{2} \mathrm{BH}_{2}\right)$ ], 并用 $\mathrm{X}$ 射线衍射对其进行了表征. 使用二甲 基正辛胺( $\mathrm{FA} /$ 胺, $m: m=11: 10$ )代替三乙胺作为溶剂, 在 $80{ }^{\circ} \mathrm{C}$ 时 $3 \mathrm{~h} \mathrm{TON}$ 达到 626. 用二甘醇或 1,4-二恶烷 代替碳酸丙烯酯, 催化剂活性降低 $65 \%$. 当催化剂的 溴、甲酸和硼烷衍生物在 $60{ }^{\circ} \mathrm{C}$ 的碳酸丙烯酯中进行 FA 脱氢时, TON 分别下降到初始值的 $85 \%$ 、39\%和 36\%.

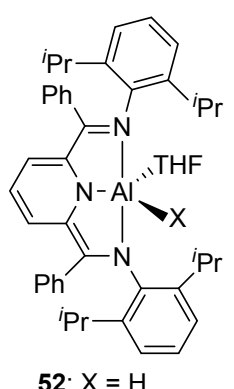

52: $\mathrm{X}=\mathrm{H}$ 53: $X=\mathrm{Me}$

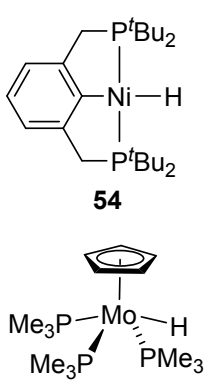

55
图 14 铝、镍和钼催化剂

Figure 14 Aluminum, nickel and molybdenum catalyst

2014 年, Zaccheria 等 ${ }^{[66]}$ 研究了 FA/胺混合物中 $\mathrm{Cu}(\mathrm{OAc})_{2} 、 \mathrm{Cu}(\mathrm{OOCH})_{2} 、 \mathrm{Cu}(\mathrm{acac})_{2} 、 \mathrm{Cu}\left(\mathrm{NO}_{3}\right)_{2} 、 \mathrm{CuO}$ 等 铜催化剂的甲酸脱氢. 除了 $\mathrm{CuCl}_{2}$ 和铜粉外, 其他的前 体几乎都没有活性. 在 $95{ }^{\circ} \mathrm{C}$ 条件下 $\mathrm{Cu}(\mathrm{OAc})_{2}$ 含量为 $0.93 \%$ 时, 三丁胺取代三乙胺使 FA 转化率从 $17.2 \%$ 提高 到 $25.6 \%$. 使用不易还原的铜前体，如 CuI，进一步增强 了 $\mathrm{FA} / \mathrm{NEt}_{3}(1: 1)$ 脱氢, 反应 $45 \mathrm{~h}$ 后最大转化率达到 $66 \%$, 以及 $\mathrm{TON}$ 为 72 .

2015 年, Neary 和 Parkin ${ }^{[67]}$ 研究了一系列钿络合物 $\left[\mathrm{Cp}^{\mathrm{R}} \mathrm{Mo}\left(\mathrm{PMe}_{3}\right)_{3-x}(\mathrm{CO})_{x} \mathrm{H}\right]\left[\mathrm{Cp}^{\mathrm{R}}=\mathrm{Cp}\right.$ (环戊二烯基), $\mathrm{Cp}^{*}$; 
表 1 部分甲酸分解制氢均相催化剂的性能

Table 1 Performance of some homogeneous catalysts for hydrogen production from formic acid decomposition

\begin{tabular}{|c|c|c|c|c|c|c|c|}
\hline Compd. & Solution $^{a}$ & Additive & $\theta /{ }^{\circ} \mathrm{C}$ & $t / \mathrm{h}$ & TON & $\mathrm{TOF}^{b} / \mathrm{h}^{-1}(t / \mathrm{min})$ & Ref. \\
\hline 4 & $\mathrm{H}_{2} \mathrm{O}$ & $\mathrm{HCO}_{2} \mathrm{Na}$ & 120 & - & - & 670 & {$[10]$} \\
\hline 5 & DMF & $\mathrm{NEt}_{3}$ & 40 & 3 & 459 & - & [16] \\
\hline 7 & $\mathrm{C}_{4} \mathrm{H}_{8} \mathrm{O}_{2}+\mathrm{H}_{2} \mathrm{O}$ & $\mathrm{HCO}_{2} \mathrm{Na}$ & 69 & 5 & 990 & $286(120)$ & [31] \\
\hline 8 & $\mathrm{C}_{4} \mathrm{H}_{8} \mathrm{O}_{2}+\mathrm{H}_{2} \mathrm{O}$ & $\mathrm{HCO}_{2} \mathrm{Na}$ & 68 & 4 & 1000 & $430(120)$ & [31] \\
\hline 9 & $\mathrm{DMF}$ & $\mathrm{NEt}_{3}$ & 90 & - & 706500 & 256000 & {$[34]$} \\
\hline 10 & DMSO & - & 50 & - & 9800 & - & [35] \\
\hline 11 & DMSO & - & 50 & - & 2400 & - & [35] \\
\hline 12 & DMSO & $\mathrm{NEt}_{3}$ & 90 & 150 & 1100000 & 7333 & {$[35]$} \\
\hline 13 & $\mathrm{H}_{2} \mathrm{O}$ & $\mathrm{HCO}_{2} \mathrm{Na}$ & 25 & - & - & 426 & [36] \\
\hline 14 & $\mathrm{H}_{2} \mathrm{O}$ & $\mathrm{HCO}_{2} \mathrm{Na}$ & 25 & - & - & $1880(10)$ & [37] \\
\hline 15 & $\mathrm{H}_{2} \mathrm{O}$ & / & 90 & 1.5 & - & 14000 & [38] \\
\hline 21 & - & $\mathrm{KHCO}_{3}$ & 60 & 4 & 5000 & $2400(5 \sim 15)$ & [39] \\
\hline 22 & - & $\mathrm{KHCO}_{3}$ & 90 & 7 & 165000 & $228000(5 \sim 15)$ & [39] \\
\hline 23 & - & $\mathrm{KHCO}_{3}$ & 60 & 1.5 & 10000 & $32000(5 \sim 15)$ & [39] \\
\hline 24 & $\mathrm{H}_{2} \mathrm{O}$ & $\mathrm{HCO}_{2} \mathrm{Na}$ & 80 & 8 & 11000 & $39500(5)$ & [40] \\
\hline 25 & $\mathrm{H}_{2} \mathrm{O}$ & - & 80 & 0.5 & 10000 & $34000(10)$ & [41] \\
\hline 26 & - & $\mathrm{HCO}_{2} \mathrm{Na}$ & 100 & 0.5 & 68000 & $322000(10)$ & [42] \\
\hline 27 & $\mathrm{H}_{2} \mathrm{O}$ & - & 60 & 2.5 & 20000 & $13300(10)$ & [43] \\
\hline 28 & $\mathrm{H}_{2} \mathrm{O}$ & - & 98.8 & 6.5 & 320000 & $62900(30)$ & [44] \\
\hline 29 & - & $\mathrm{HCO}_{2} \mathrm{Na}$ & 90 & 35 & 350000 & $12000(10)$ & [45] \\
\hline 30 & ${ }^{t} \mathrm{BuOH}$ & $\mathrm{NEt}_{3}$ & 80 & 0.016 & 2000 & $120000(1)$ & [48] \\
\hline 31 & $\mathrm{H}_{2} \mathrm{O}$ & $\mathrm{NaHCO}_{3}$ & 50 & 1 & 20 & $20(60)$ & {$[50]$} \\
\hline 32 & $\mathrm{H}_{2} \mathrm{O}$ & $\mathrm{NaHCO}_{3}$ & 50 & 1 & 11 & $11(60)$ & {$[50]$} \\
\hline 33 & $\mathrm{H}_{2} \mathrm{O}$ & $\mathrm{NaHCO}_{3}$ & 50 & 1 & 130 & $130(60)$ & {$[50]$} \\
\hline 34 & $\mathrm{H}_{2} \mathrm{O}$ & $\mathrm{NaHCO}_{3}$ & 50 & 48 & 7850 & $650(60)$ & {$[50]$} \\
\hline 35 & $\mathrm{H}_{2} \mathrm{O}$ & - & 60 & 0.33 & 7750 & $28750(3)$ & [51] \\
\hline 36 & $\mathrm{H}_{2} \mathrm{O}$ & - & 60 & 0.33 & 8333 & $33750(3)$ & [51] \\
\hline 37 & $\mathrm{H}_{2} \mathrm{O}$ & - & 60 & 0.33 & 8167 & $31250(3)$ & [51] \\
\hline 38 & $\mathrm{H}_{2} \mathrm{O}$ & $\mathrm{HCO}_{2} \mathrm{Na}$ & 20 & 7 & 20 & 28 & [52] \\
\hline 39 & - & $\mathrm{NEt}_{3}+\mathrm{KI}$ & 60 & 0.5 & 706 & $4375(3 \sim 6)$ & [53] \\
\hline 40 & $\mathrm{C}_{4} \mathrm{H}_{8} \mathrm{O}_{2}$ & - & 75 & 16 & 1024 & $169(10)$ & [54] \\
\hline 41 & $\mathrm{H}_{2} \mathrm{O}$ & - & 100 & 50 & 449000 & 39200 (180) & [55] \\
\hline 42 & DMF & $\mathrm{NEt}_{3}$ & 60 & 3 & 19 & 200 & [56] \\
\hline 43 & DMF & $\mathrm{NEt}_{3}$ & 60 & 15 & 702 & $138(180)$ & [57] \\
\hline 44 & $\mathrm{PC}$ & - & 80 & 16 & 92000 & 9425 & [58] \\
\hline 45 & THF & $\mathrm{NEt}_{3}$ & 40 & 3 & $>999$ & $500(60)$ & [32] \\
\hline 46 & THF & $\mathrm{NEt}_{3}$ & 40 & 3 & $>999$ & $503(60)$ & [32] \\
\hline 47 & THF & $\mathrm{NEt}_{3}$ & 40 & 3 & $>999$ & $393(60)$ & [32] \\
\hline 48 & $\mathrm{C}_{4} \mathrm{H}_{8} \mathrm{O}_{2}$ & $\mathrm{Li}^{\prime} \mathrm{BF}_{4}$ & 80 & 9.5 & 983642 & $196728(60)$ & [61] \\
\hline 49 & $\mathrm{C}_{4} \mathrm{H}_{8} \mathrm{O}_{2}$ & $\mathrm{NEt}_{3}$ & 80 & 2.5 & 994 & $739(10)$ & [61] \\
\hline 50 & $\mathrm{PC}$ & - & 60 & 6 & 6061 & $1737(10)$ & [62] \\
\hline 51 & $\mathrm{PC}$ & $\mathrm{NEt}_{3}$ & 80 & 6 & 10000 & $2635(60)$ & [63] \\
\hline 52 & THF & $\mathrm{NEt}_{3}$ & 65 & 1 & 2200 & $5200(15)$ & [64] \\
\hline 53 & THF & $\mathrm{NEt}_{3}$ & 65 & 1 & 2000 & $4800(15)$ & [64] \\
\hline 54 & $\mathrm{PC}$ & $n \mathrm{OctNMe}_{2}$ & 80 & 3 & 626 & $481(120)$ & [65] \\
\hline 55 & $\mathrm{C}_{6} \mathrm{H}_{6}$ & - & 100 & - & - & 31 & [67] \\
\hline
\end{tabular}

${ }^{a}$ All solutions add formic acid by default; ${ }^{b}$ The time corresponding to TOF is in brackets.

$x=0,1,2,3]$, 在无碱条件下催化甲酸脱氢. 在杂化络合 物 $\left[\mathrm{CpMo}\left(\mathrm{PMe}_{3}\right)_{2}(\mathrm{CO}) \mathrm{H}\right](\mathbf{5 5})$ 存在的情况下，以苯作为 溶剂, 反应温度为 $100{ }^{\circ} \mathrm{C}$ 时, 氢的反应速率可以达到 31 $\mathrm{h}^{-1}$. 采用空间位阻更大的 $\mathrm{Cp}$ *配体时, TOF 略有增加到 $54 \mathrm{~h}^{-1}$. 而 $\left[\mathrm{CpMo}\left(\mathrm{PMe}_{3}\right)(\mathrm{CO})_{2} \mathrm{H}\right]$ 和 $\left[\mathrm{CpMo}(\mathrm{CO})_{3} \mathrm{H}\right]$ 对于
甲酸脱氢反应不具有催化活性，可以被理解为它们不容 易被甲酸质子化. 在随后的一项研究中，作者报道了镍 络合物 $\left[\mathrm{Ni}\left(\mathrm{PMe}_{3}\right)_{4}\right]$ 在苯中 $80{ }^{\circ} \mathrm{C}$ 无添加碱条件下催化甲 酸脱氢, TOF 为 $0.2 \mathrm{~h}^{-1}, 14 \mathrm{~h}$ 后的 TON 为 $70^{[68]}$. 


\section{3 结论}

人类对可持续和可再生能源的不断追求将主导未 来的能源研究. 如果能克服氢气安全高效储存的瓶颈, 氢气将成为未来广泛使用的能源. 甲酸/甲酸盐具有作 为液氢储存材料和清洁制氢的巨大潜力. 在过去的十几 年中, 人们不仅在开发活性更强、更稳定、选择性更强 的催化剂方面取得了重大进展, 而且提出了 $\mathrm{H}_{2}$ 释放和 储存循环系统的概念. 例如, Himeda 开发的碱性催化剂 可以在室温和常压下催化甲酸水溶液中的可逆氢生成. 此外, Laurenczy 的水溶性 $\mathrm{Ru}$ 催化剂在高效制氢和储存 氢气方面也很有价值. 除水外, 有机介质(溶剂、胺)和双 相介质(溶剂、水)也为甲酸高效催化释氢提供了基础. 含有螯合型、质子反应性和多齿配体的催化剂体系也备 受科研工作者的关注. 除了钌、铑和铱等贵金属催化剂, 包括铁、钴、镍、铜和铝等非贵金属催化剂也逐渐发展 起来, 尤其是铁基催化剂体系, Milstein 从胺和无胺的甲 酸溶液改进了铁催化产氢的效率. 与此同时, 近年来对 甲酸脱氢催化机理也有了更为详细的了解.

然而, 在这一领域仍然存在一些挑战, 例如: 只有 少数催化剂体系在较温和的反应条件下仍然具有良好 的催化性能, 当反应条件比较恶劣时, 催化剂往往会存 在失活或者活性降低的现象. 铁基催化剂体系的配体范 围有限, 还需要开发稳定的、合适的非贵金属催化剂. 目前, 很多金属催化剂需要有碱添加剂的情况才表现出 高活性，但这导致了实际氢气充放电装置的构造复杂 化, 未来储氢系统的特点应该是简单和环境兼容性, 要 求没有添加剂和有机溶剂, 依然实现氢气高效存储和释 放. 自 2008 年 Laurenczy 和 Beller 提出二氧化碳和氢气 可逆循环系统的概念以来, 在脱氢和加氢这两个半反应 的结合方面取得了较大进展. 除了开发合适的脱氢/加 氢催化剂外, 如何控制这两种半反应显得至关重要. 而 且, 为了建立实用的可逆储氢体系, 还需要解决可回收 性和能源效率方面的问题. 此外，甲醇、乙醇、甘油，以 及从生物质衍生的复杂多元醇, 如葡萄糖、果糖和纤维 二糖等成功制取氢气, 也为未来氢能源开发提供了多样 化途径.

\section{References}

[1] Sordakis, K.; Tang, C.; Vogt, L. K.; Junge, H.; Dyson, P. J.; Beller, M.; Laurenczy, G. Chem. Rev. 2018, 118, 372.

[2] Coffey, R. S. Chem. Commun. 1967, 923.

[3] Gao, Y.; Kuncheria, J.; Puddephatt, R. J.; Yap, G. P. A. Chem. Commun. 1998, 2365.

[4] Gao, Y.; Kuncheria, J. K.; Jenkins, H. A.; Puddephatt, R. J.; Yap, G. P. A. J. Chem. Soc., Dalton Trans. 2000, 3212.

[5] Hannedouche, J. M.; Clarkson, G. J.; Wills, M. J. Am. Chem. Soc. 2004, 126, 986 .

[6] Hayes, A. M.; Morris, D. J.; Clarkson, G. J.; Wills, M. J. Am. Chem. Soc. 2005, 127, 7318.
[7] Matharu, D. S.; Morris, D. J.; Clarkson, G. J.; Wills, M. Chem. Commun. 2006, 3232.

[8] Morris, D. J.; Clarkson, G. J.; Wills, M. Organometallics 2009, $28,4133$.

[9] Majewski, A.; Morris, D. J.; Kendall, K.; Wills, M. ChemSusChem 2010, 3, 431.

[10] Fellay, C.; Paul; nbsp; Dyson, J.; Laurenczy, G. Angew. Chem. Int. Ed. 2008, 120, 4030.

[11] Fellay, C.; Yan, N.; Dyson, P. J.; Laurenczy, G. Chem.-Eur. J. 2009, 15, 3752 .

[12] Thevenon, A.; Frost-Pennington, E.; Weijia, G.; Dalebrook, A. F.; Laurenczy, G. ChemCatChem 2014, 6, 3146.

[13] Gan, W.; Fellay, C.; Dyson, P. J.; Laurenczy, G. J. Coord. Chem. 2010, 63, 2685.

[14] Gan, W.; Snelders, D. J. M.; Dyson, P. J.; Laurenczy, G. B. Chem CatChem 2013, 5, 1126.

[15] Loges, B.; Boddien, A.; Junge, H.; Beller, M. Angew. Chem., Int. Ed. 2008, 47, 3962.

[16] Boddien, A.; Loges, B. R.; Junge, H.; Beller, M. ChemSusChem 2008, 1, 751 .

[17] Boddien, A.; Loges, B.; Junge, H.; Gärtner, F.; Beller, M. Adv. Synth. Catal. 2009, 351, 2517.

[18] Boddien, A.; Loges, B.; Junge, H.; Noyes, J. R.; Beller, M. Tetrahedron Lett. 2009, 50, 1603.

[19] Loges, B.; Boddien, A.; Junge, H.; Noyes, J. R.; Baumann, W.; Beller, M. Chem. Commun. 2009, 4185.

[20] Boddien, A.; Federsel, C.; Sponholz, P.; Mellmann, D.; Jackstell, R.; Junge, H.; Laurenczy, G.; Beller, M. Energy Environ. Sci. 2012, 5, 8907.

[21] Sordakis, K.; Beller, M.; Laurenczy, G. ChemCatChem 2014, 6, 96.

[22] Sponholz, P.; Mellmann, D. r.; Junge, H.; Beller, M. ChemSusChem 2013, 6, 1172 .

[23] Geldbach, T. J.; Dyson, P. J. J. Am. Chem. Soc. 2004, 126, 8114.

[24] Kawasaki, I.; Tsunoda, K.; Tsuji, T.; Yamaguchi, T.; Shibuta, H.; Uchida, N.; Yamashita, M.; Ohta, S. Chem. Commun. 2005, 2134.

[25] Li, X.; Ma, X.; Shi, F.; Deng, Y. ChemSusChem 2010, 3, 71.

[26] Scholten, J. D.; Prechtl, M. H. G.; Dupont, P. Â. J. ChemCatChem 2010, 2, 1265

[27] Berger, M. E. M.; Assenbaum, D.; Taccardi, N.; Spiecker, E.; Wasserscheid, P. Green Chem. 2011, 13, 1411.

[28] Schuster, O.; Yang, L.; Raubenheimer, H. G.; Albrecht, M. Chem. Rev. 2009, 109, 3445.

[29] Czaun, M.; Goeppert, D. A.; May, R.; Haiges, D. R.; Prakash, P. D. G. K. S.; Olah, P. D. G. A. ChemSusChem 2011, 4, 1241.

[30] Czaun, M.; Goeppert, A.; Kothandaraman, J.; May, R. B.; Olah, G. A. ACS Catal. 2013, 4, 311 .

[31] Kothandaraman, J.; Czaun, M.; Goeppert, A.; Haiges, R.; Jones, J.-P.; May, R. B.; Prakash, G. K. S.; Olah, G. A. ChemSusChem 2015, 8,1442

[32] Zell, T.; Butschke, B.; Ben-David, Y.; Milstein, D. Chem.-Eur. J. 2013, 19, 8068 .

[33] Vogt, M.; Nerush, A.; Diskin-Posner, Y.; Ben-David, Y.; Milstein, D. Chem. Sci. 2014, 5, 2043.

[34] Filonenko, G. A.; van Putten, R.; Schulpen, E. N.; Hensen, E. J. M.; Pidko, E. A. ChemCatChem 2014, 6, 1526.

[35] Pan, Y.; Pan, C.-L.; Zhang, Y.; Li, H.; Min, S.; Guo, X.; Zheng, B.; Chen, H.; Anders, A.; Lai, Z.; Zheng, J.; Huang, K.-W. Chem. Asian J. 2016, 11, 1357.

[36] Fukuzumi, S.; Kobayashi, T.; Suenobu, T. J. Am. Chem. Soc. 2010, 132, 1496.

[37] Maenaka, Y.; Suenobu, T.; Fukuzumi, S. Energy Environ. Sci, 2012, 5, 7360 .

[38] Himeda, Y. Green Chem. 2009, 11, 2018.

[39] Hull, J. F.; Himeda, Y.; Wang, W.-H.; Hashiguchi, B.; Periana, R.; Szalda, D. J.; Muckerman, J. T.; Fujita, E. Nat. Chem. 2012 4,383 . 
[40] Wang, W. H.; Xu, S.; Manaka, Y.; Suna, Y.; Kambayashi, H.; Muckerman, J. T.; Fujita, E.; Himeda, Y. ChemSusChem 2014, 7, 1976.

[41] Manaka, Y.; Wang, W. H.; Suna, Y.; Kambayashi, H.; Muckerman, J. T.; Fujita, E.; Himeda, Y. Catal. Sci. Technol. 2013, 4, 34.

[42] Wang, W.-H.; Ertem, M. Z.; Xu, S.; Onishi, N.; Manaka, Y.; Suna, Y.; Kambayashi, H.; Muckerman, J. T.; Fujita, E.; Himeda, Y. ACS Catal. 2015, 5, 5496.

[43] Onishi, N.; Ertem, M. Z.; Xu, S.; Tsurusaki, A.; Manaka, Y.; Muckerman, J. T.; Fujita, E.; Himeda, Y. Catal. Sci. Technol. 2016, 6.

[44] Iguchi, M.; Himeda, Y.; Manaka, Y.; Kawanami, H. ChemSusChem 2016, 9, 2749.

[45] Guan, C.; Zhang, D.-D.; Pan, Y.; Iguchi, M.; Ajitha, M. J.; Hu, J.; Li, H.; Yao, C.; Huang, M.-H.; Min, S.; Zheng, J.; Himeda, Y.; Kawanami, H.; Huang, K.-W. Inorg. Chem. 2017, 56, 438.

[46] Iguchi, M.; Himeda, Y.; Manaka, Y.; Matsuoka, K.; Kawanami, H. ChemCatChem 2016, 8,886 .

[47] Barnard, J. H.; Wang, C.; Berry, N. G.; Xiao, J. Chem. Sci. 2013, 4, 1234.

[48] Tanaka, R.; Yamashita, M.; Chung, L. W.; Morokuma, K.; Nozaki, K. Organometallics 2011, 30, 6742.

[49] Fink, C.; Laurenczy, G. Dalton. Trans. 2017, 46, 1670.

[50] Onishi, N.; Kanega, R.; Fujita, E.; Himeda, Y. Adv. Synth. Catal. 2019, 361, 289.

[51] Lu, S.-M.; Wang, Z.; Wang, J.; Li, J.; Li, C. Green Chem. 2018, $20,1835$.

[52] Fukuzumi, S.; Kobayashi, T.; Suenobu, T. ChemSusChem 2008, $1,827$.

[53] Wang, Z.; Lu, S.-M.; Wu, J.; Li, C.; Xiao, J. Eur. J. Inorg. Chem. 2016, 2016, 490.

[54] Jongbloed, L.; Bruin, B. D.; Reek, J. N. H.; Lutz, M.; Vlugt, J. I.
V. D. Catal. Sci. Technol. 2015, 6, 1320.

[55] Jantke, D.; Pardatscher, L.; Drees, M.; Cokoja, M.; Herrmann, W. A.; Kühn, F. E. ChemSusChem 2016, 9, 2849.

[56] Boddien, A.; Loges, B.; Gärtner, F.; Torborg, C.; Fumino, K.; Junge, H.; Ludwig, R.; Beller, M. J. Am. Chem. Soc. 2010, 132 , 8924.

[57] Boddien, A.; Gärtner, F.; Jackstell, R.; Junge, H.; Spannenberg, A.; Baumann, W.; Ludwig, R.; Beller, M. Angew. Chem., Int. Ed. 2010, 49, 8993.

[58] Boddien, A.; Mellmann, D.; Gärtner, F.; Jackstell, R.; Junge, H.; Dyson, P. J.; Laurenczy, G.; Ludwig, R.; Beller, M. Science 2011, 333,1733

[59] Mellmann, D. R.; Barsch, E.; Bauer, M.; Grabow, K.; Boddien, A.; Kammer, A.; Sponholz, P.; Bentrup, U.; Jackstell, R.; Junge, H. Chem.-Eur. J. 2014, 20, 13589.

[60] Montandon-Clerc, M.; Dalebrook, A. F.; Laurenczy, G. J. Catal. 2016, 343, 62

[61] Bielinski, E. A.; Lagaditis, P. O.; Zhang, Y.; Mercado, B. Q.; Würtele, C.; Bernskoetter, W. H.; Hazari, N.; Schneider, S. J. Am. Chem. Soc. 2014, 136, 10234.

[62] Bertini, F.; Mellone, I.; Ienco, A.; Peruzzini, M.; Gonsalvi, L. ACS Catal. 2015, 5, 1254.

[63] Mellone, I.; Gorgas, N.; Bertini, F.; Peruzzini, M.; Kirchner, K.; Gonsalvi, L. Organometallics 2016, 35, 3344.

[64] Myers, T. W.; Berben, L. A. Chem. Sci. 2014, 5, 2771.

[65] Enthaler, S.; Brück, A.; Kammer, A.; Junge, H.; Gülak, S. ChemCatChem 2014, 7, 65.

[66] Scotti, N.; Psaro, R.; Ravasio, N.; Zaccheria, F. RSC Adv. 2014 4, 61514 .

[67] Neary, M. C.; Parkin, G. Chem. Sci. 2015, 6, 1859.

[68] Neary, M. C.; Parkin, G. Dalton. Trans. 2016, 45, 14645. 